Methods Using ICD 10 codes for SLE (M32, M320, M321, M328, M329), we identified reproductive aged (ages 1650) female SLE patients, who presented to the outpatient rheumatology clinic at Parkland Hospital between 07/01/17 and 10/31/17. We performed a retrospective chart review of the documentation of contraception use. We also assessed medication use including potential teratogenic medications.

Results Among 112 clinic encounters, contraceptive use was documented in $60 \%$ of encounters. Amongst those women prescribed a potentially teratogenic medication, documentation of contraception was present in $68 \%$ of clinic notes. In our patient population, the most commonly prescribed potentially teratogenic medication was mycophenolate mofetil followed by methotrexate. The average age of women who were on potentially teratogenic medicine and had documented contraception was younger (mean 31, SD 7.2) than those in whom contraception was not documented (mean 38, SD 8.6). Greater than 50\% of those patients on potentially teratogenic medicine used less reliable contraceptive methods: abstinence or condoms. Providers rarely documented other information in regard to reproductive health such as pregnancy planning and the desire to have children.

Conclusions Contraceptive use documentation amongst reproductive aged women with SLE in a safety net hospital is often lacking even amongst those on potentially teratogenic medication. Documentation of contraception is more common in younger women. Identifying barriers to contraceptive use documentation and implementing interventions to facilitate documentation is a future goal.

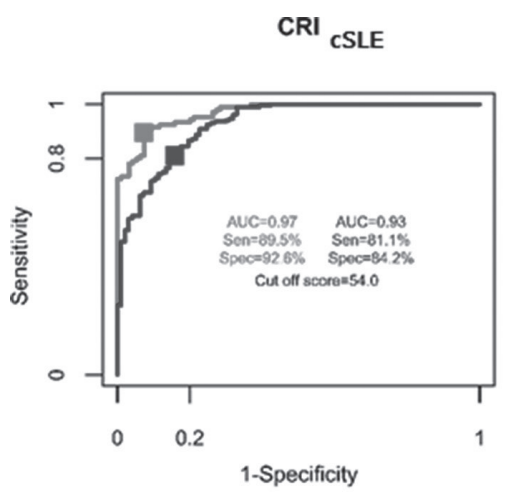

At Least Moderate Improvement

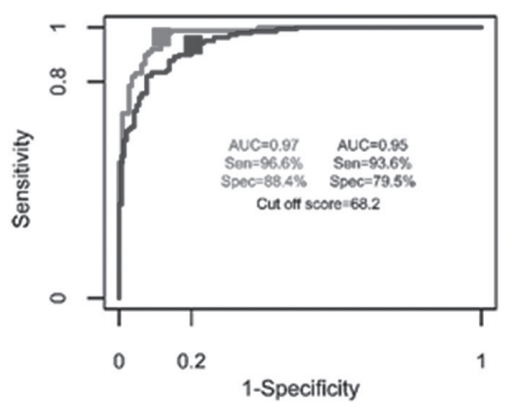

\section{CS-10 CRITERIA FOR CLINICALLY RELEVANT IMPROVEMENT IN CHILDREN \& ADOLESCENTS WITH CHILDHOOD-ONSET SYSTEMIC LUPUS ERYTHEMATOSUS}

${ }^{1}$ Hermine I Brunner*, ${ }^{1}$ Michael Holland, ${ }^{2}$ Michael W Beresford, ${ }^{3}$ Stacy P Ardoin, ${ }^{4}$ Simone Appenzeller, ${ }^{5}$ Clovis A Silva, ${ }^{6}$ Francisco Flores, ${ }^{7}$ Beatrice Goilav, ${ }^{1}$ Pinar Ozge Avar, ${ }^{8}$ Scott E Wenderfer, ${ }^{9}$ Deborah M Levy, ${ }^{10}$ Angelo Ravelli, ${ }^{11}$ Raju Khunchandani, ${ }^{12}$ Tadej Avcin, ${ }^{13}$ Marisa S Klein-Gitelman, ${ }^{9}$ Brian M Feldman, ${ }^{10}$ Nicola Ruperto, ${ }^{14} \mathrm{Jun}$ Ying. ${ }^{1}$ Department of Pediatrics, University of Cincinnati and Division of Rheumatology, Cincinnati Children's Hospital Medical Center, Cincinnati, OH; ${ }^{2}$ Institute of Translational Medicine, University of Liverpool, Liverpool, UK; Department of Paediatric Rheumatology, Alder Hey Children's NHS Foundation Trust, Liverpool, UK; ${ }^{3}$ Department of Pediatrics and Internal Medicine, Ohio State University, Division of Rheumatology, Nationwide Children's Hospital and Wexner Medical Center, Columbus, OH; ${ }^{4}$ Rheumatology Unit-Faculty of Medical Science; University of Campinas, Campinas, Brazil; ${ }^{5}$ Children's Institute, Hospital das Clinicas HCFMUSP; Faculdade de Medicina da Universidade de São Paulo, Brazil; ${ }^{6}$ Department of Pediatrics, University of Cincinnati and Division of Nephrology and Hypertension, Cincinnati Children's Hospital Medical Center, Cincinnati, OH; ${ }^{7}$ The Children's Hospital at Montefiore, Division of Nephrology and Department of Pediatrics, Albert Einstein College of Medicine, Bronx, NY; ${ }^{8}$ Department of Pediatrics, Baylor College of Medicine; Renal Section, Texas Children's Hospital Houston, TX; ${ }^{9}$ Department of Pediatrics, University of Toronto and Division of Rheumatology, Hospital for Sick Children, Toronto, Canada; ${ }^{10}$ Istituto G. Gaslini, Pediatria II, Reumatologia, Genoa, Italy; ${ }^{11}$ Pediatric Rheumatology; Jaslok Hospital Mumbai, India; ${ }^{12}$ Department of Allergology, Rheumatology and Clinical Immunology, University Children's Hospital, University Medical Centre Ljubljana, Slovenia; ${ }^{13}$ Department of Pediatrics, Northwestern University Feinberg School of Medicine and Division of Rheumatology, Ann and Robert Lurie Children's Hospital of Chicago, Chicago, IL;

${ }^{14}$ Department of Environmental Health Sciences, University of Cincinnati, Cincinnati, $\mathrm{OH}$

\subsection{6/lupus-2018-Ism.45}

Background There is international consensus around a core set of variables (cSLE-CRVs) to assess response to therapy with childhood-onset systemic lupus erythematosus (cSLE) [global assessment of patient well-being (Patient-global), physician

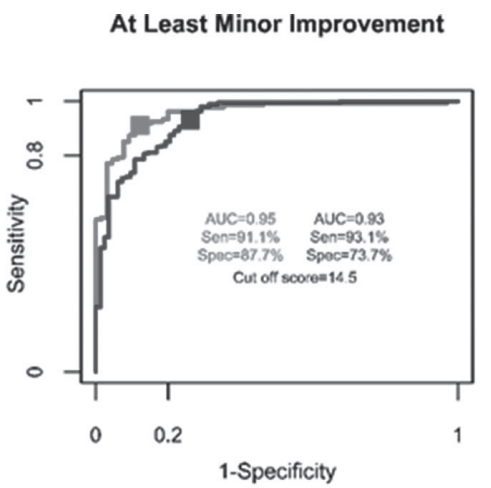

Major Improvement

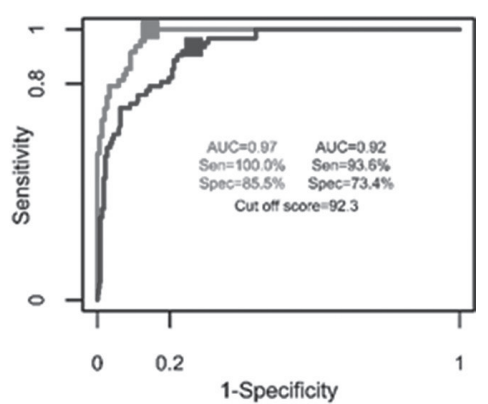

Abstract CS-10 Figure 1 CHILI scores were calculated from logit scores using multivariate logistic models considering either absolute differences between the baseline and follow-up time point at the time of follow-up relative of the baseline visit in the trainings-dataset. The range of the improvement score has been standardized to range from 0-100, the higher (lower) score indicates higher (lower) likelihood of improvement. The CHILI score $=100 \times$ raw score/ $(1+$ raw score $)$. The raw score is calculated as follows: raw score $=\operatorname{Exp}(5.1+0.47 \times S L E D A I+0.7 \times M D-g l o b a l+1.1 \times P C-$ ratio- 0.32 xpatient-global $+0.002 \times C H Q-P h s$ ) 
assessment of cSLE activity (MD-global), disease activity index score, urine protein to creatinine ratio (PCR), Child Health Questionnaire physical function summary score (CHQ-Phs)]. Percentage changes of these cSLE-CRVs are used in the Provisional PRINTO-ACR- EULAR Criteria of Response to Therapy of cSLE (PCI). In a small dataset, we have previously shown that the PCI and the Systemic Lupus Responder Index only have fair accuracy in detecting cSLE improvement. The Objective of this research was to 1) validate the PCI and 2) develop for Children an Index of Lupus Improvement (CHILI) as a tool to measure response to therapy, with focus on clinically relevant improvement (CRI $\mathrm{CSLE})$.

Methods Pediatric subspecialists $(n=213)$ in treating cSLE were invited to define $\mathrm{CRI}_{\mathrm{CSLE}}$ and rate a total of 433 unique patient profiles for the presence/absence of $\mathrm{CRI}_{\mathrm{CSLE}}$ and various levels of improvement. Patient profiles included the cSLECRVs and routine laboratory tests at a baseline and follow-up time-point. To measure $\mathrm{CRI}_{\mathrm{CSLE}}$ we tested the PCI, and developed the candidate CHILI criteria that considered a) absolute and b) percentage changes of the cSLE-CRVs (baseline vs follow-up) in a trainings-dataset and initially validated these criteria in the validation-dataset. Criteria accuracy was assessed by kappa statistics (PCI) and the area under the ROC curve (AUC; range: 0-1)], respectively.

Results During an international consensus conference agreement on a definition of $\mathrm{CRI}_{\mathrm{cSLE}}$ was achieved. Response rate to patient profile ratings was 91\% (194/214). The PIC had no more than fair accuracy (kappa <0.43). There was consensus (92.3\%) that the CHILI is preferable to the PCI to measure $\mathrm{CRI}_{\mathrm{CSLE}}$. As shown in the figure 1, considering absolute changes of the cSLE-CRVs, a CHILI score of $\geq 54$ (range 0100) was highly accurate for identifying $\mathrm{CRI}_{\mathrm{CSLE}}$ (AUC=0.93; sensitivity $=81.1 \%$; specificity $=84.2 \%$ ) in the validation-dataset. Likewise CHILI scores exceeding 15, 68 and 92 reflected minor, moderate and major improvement for values (all: AUC >0.92, sensitivity: >93.1\%; specificity: >73.4\%), respectively.

Conclusions The CHILI is a new highly accurate index to capture improvement in the overall course of cSLE. This index is also useful to categorize the degree of cSLE response to therapy. Acknowledgements For the Pediatric Rheumatology International Trial Network and the Pediatric Rheumatology Collaborative Study Group; the study is supported by NIH grants 5U01-AR51868, P30-AR AR47363 and 2UL1RR026314 and the PRCSG and PRINTO Coordinating Centers. This study is also supported by grants from Fundação de Amparo à Pesquisa do Estado de São Paulo (FAPESP 2015/03756-4 to CAS), Conselho Nacional de Desenvolvimento Científico e Tecnológico (CNPq 303422/2015-7 to CAS) and by Núcleo de Apoio à Pesquisa 'Saúde da Criança e do Adolescente' da USP (NAP-CriAd) to CAS.

\section{CS-11 THE EFFECTS OF CONTEXTUAL SOCIODEMOGRAPHIC FACTORS AND HEALTH RESOURCE CONCENTRATION ON HYDROXYCHLOROQUINE ADHERENCE AMONG U.S. MEDICAID BENEFICIARIES WITH LUPUS}

${ }^{1}$ Candace H Feldman* ${ }^{1}$ Karen H Costenbader, ${ }^{1}$ Daniel H Solomon, ${ }^{2}$ SV Subramanian, ${ }^{2}$ Ichiro Kawachi. 'Division of Rheumatology, Immunology and Allergy, Department of Medicine, Brigham and Women's Hospital, Boston, MA, USA; ${ }^{2}$ Department of Social and Behavioral Sciences, Harvard T.H. Chan School of Public Health, Boston, MA, USA

10.1136/lupus-2018-Ism.46
Background Adherence to hydroxychloroquine (HCQ), the standard-of-care treatment among patients with systemic lupus erythematous (SLE) is suboptimal and interventions to date to improve this have been largely unsuccessful. Individual-level factors, including younger age and non-white race/ethnicity, have been implicated. While contextual influences such as area-level poverty are known to contribute to health behaviors and to SLE-related damage, no studies to date investigate the role of these factors on HCQ adherence. We therefore aimed to use multilevel models to understand the effects of sociodemographic variables (area-level racial composition, poverty, educational attainment) and health resource concentration (numbers of physicians, pharmacists, hospitals) on HCQ adherence while also accounting for potential individual-level factors.

\begin{tabular}{|c|c|c|}
\hline Area-level sociodemographics and health resources & $\begin{array}{l}\text { Odds ratio } \\
\text { (OR) }\end{array}$ & $\begin{array}{l}95 \% \text { Credible } \\
\text { Intervals }\end{array}$ \\
\hline \multicolumn{3}{|l|}{ Zip Code-level Percent Black (ref=lowest tertile) } \\
\hline Tertile 2 & 0.85 & $0.74-0.98$ \\
\hline Tertile 3 & 0.81 & $0.69-0.96$ \\
\hline \multicolumn{3}{|l|}{ Zip Code-level Percent White (ref=lowest tertile) } \\
\hline Tertile 2 & 0.93 & $0.81-1.08$ \\
\hline Tertile 3 & 1.13 & $0.94-1.34$ \\
\hline \multicolumn{3}{|l|}{ Zip Code-level Percent Hispanic (ref=lowest tertile) } \\
\hline Tertile 2 & 0.92 & $0.77-1.07$ \\
\hline Tertile 3 & 0.90 & $0.74-1.06$ \\
\hline \multicolumn{3}{|l|}{ Zip Code-level Percent below Federal Poverty Level } \\
\hline \multicolumn{3}{|l|}{ (FPL) (ref=lowest tertile) } \\
\hline Tertile 2 & 0.98 & $0.83-1.16$ \\
\hline Tertile 3 & 1.02 & $0.88-1.19$ \\
\hline \multicolumn{3}{|l|}{ Zip Code-level Educational attainment (ref=HS or less) } \\
\hline Some college & 1.10 & $0.83-1.46$ \\
\hline College graduate & 1.00 & $0.83-1.19$ \\
\hline \multicolumn{3}{|l|}{ Zip Code-level Composite Percent Black/Percent } \\
\hline \multicolumn{3}{|l|}{ below $\mathrm{FPL}^{*}$ (ref=Group 1) } \\
\hline Group 2 & 0.88 & $0.73-1.04$ \\
\hline Group 3 & 0.87 & $0.70-1.07$ \\
\hline \multicolumn{3}{|l|}{ County-level number of hospitals per capita ${ }^{* *}$} \\
\hline \multicolumn{3}{|l|}{ (ref=lowest tertile) } \\
\hline Tertile 2 & 1.13 & $0.97-1.31$ \\
\hline Tertile 3 & 1.30 & $1.07-1.58$ \\
\hline \multicolumn{3}{|l|}{ State-level number of rheumatologists per capita* } \\
\hline \multicolumn{3}{|l|}{ (ref=lowest tertile) } \\
\hline Tertile 2 & 1.22 & $0.95-1.55$ \\
\hline Tertile 3 & 1.16 & $0.89-1.47$ \\
\hline \multicolumn{3}{|c|}{$\begin{array}{l}\text { Each variable was examined in a separate model adjusted for age, sex, race/ethnicity, SLE } \\
\text { risk adjustment index, lupus nephritis, diabetes, antidepressant use, corticosteroid use, } \\
\text { immunosuppressive use, number of lab tests, number of medications, healthcare utilization, } \\
\text { calendar year at index date, and random effects at each level. Bolded } 95 \% \text { credible inter- } \\
\text { vals do not cross } 1 \text { and Bayesian p-value was }<0.05 \text {. } \\
\text { "Group } 1=z i p \text { codes with the lowest tertiles of percent black and percent below FPL, Group }\end{array}$} \\
\hline \multicolumn{3}{|c|}{$\begin{array}{l}\text { 3=zip codes with the highest tertiles of } \% \text { black and } \% \text { below FPL } \\
{ }^{* *} \text { Models also adjusted for zip code percent black }\end{array}$} \\
\hline
\end{tabular}

Methods We identified SLE patients with new use of HCQ (no use in $\geq 6$ months) in Medicaid (2000-2010) from $28 \mathrm{U}$. S. states. We required 12 months of continuous enrollment after HCQ initiation with complete drug dispensing data and measured adherence using the proportion of days covered 